\title{
Redescubriendo a la persona en la adolescente madre. Retornar su mirada hacia ella misma
}

\author{
Rediscovering the Person in the Adolescent Mother: \\ Bringing her looks to herself
}

\author{
Ana Arguedas-Ramírez \\ Universidad de Costa Rica \\ San José, Costa Rica \\ ana.arguedas.ramirez@una.cr \\ https://orcid.org/0000-0002-7853-3785 \\ Manuel Rodríguez-Madrigal \\ Universidad Nacional \\ San José, Costa Rica \\ rmanull@hotmail.com \\ Alejandro Alfaro-Arguedas \\ Universidad Nacional \\ San José, Costa Rica \\ alejandro05alfa@gmail.com
}

Recibido: 03/08/2020 Aceptado: 05/11/2020

Resumen. Redescubriendo a la persona en la adolescente madre consiste en un cambio en el abordaje de esta condición, al rescatar en orden prioritario el estadio adolescente en que se encuentra la persona, en donde se generan una serie de cambios súbitos que conllevan, además, nuevas exigencias en paralelo a su adolescencia, su condición de embarazo y las responsabilidades para la joven y sus familias.

En este proceso se promueve la construcción de conocimientos y actitudes, con el fin de potenciar a la adolescente madre en su propio redescubrimiento, mediante la participación en espacios de autorreflexión, desde la visión de sí misma y como persona con derechos.

El redescubrimiento es la metodología diseñada para facilitar el aprendizaje y cambio de conductas con respecto a sí misma. Se parte de sus sentimientos, temores y dificultades como insumos básicos para la interiorización de los nuevos conocimientos, actitudes y habilidades. Esta experiencia se realizó durante cuatro años (2015-2018). El Instituto de Estudios Interdisciplinarios de la Niñez y la Adolescencia (Ineina), junto con el Hospital San Vicente de Paúl y el Patronato Nacional de la Infancia, de forma interdisciplinar, transdisciplinar e interinstitucional, lograron mancomunar esfuerzos en la generación de sus propias reflexiones en las personas, sus familias e instituciones participantes. 
ReVista Universidad en Diálogo • Vol. 11, N. ${ }^{\circ}$ 1, Enero-Junio, 2021 • 179-198

ISSN 2215-2849 • EISSN: 2215-4752

URL: http://www.revistas.una.ac.cr/index.php/dialogo/index CorReo ElECTRÓNICO: universidadendialogo@una.cr DOI: http://doi.org/10.15359/udre.11-1.7

Palabras clave: redescubrimiento, adolescencia, embarazo, potenciación, desarrollo integral, derechos humanos, vida con proyectos.

\begin{abstract}
Rediscovering the person in the adolescent mother" is a change in the approach to this condition by rescuing, in priority order, the adolescent situation in which the person is situated. It is a condition where a series of sudden changes are generated. In addition, these changes entail new demands in parallel to her adolescence, pregnancy condition, and the responsibilities for the young woman and her family.

In this process, the construction of knowledge and attitudes are promoted in order to empower the adolescent mother in her own rediscovery through her participation in spaces for self-reflection, from the vision of herself and as a person with rights.

Rediscovery is the methodology designed to ease the learning and behavior change respecting to herself. It is based on her feelings, fears, difficulties as basic inputs for internalizing new knowledge, attitudes, and skills.

This experience has been carried out for four years (2015-2018), in which the Institute for Interdisciplinary Studies on Childhood and Adolescence (INEINA), together with the San Vicente de Paúl Hospital and the Child Welfare Office (PANI), in an interdisciplinary, transdisciplinary, and inter-institutional action, achieved joint efforts in the generation of their own reflections on the people, their families, and participating institutions.
\end{abstract}

Keywords: person rediscovery, adolescence, pregnancy, empowerment, integral development, human rights, life with projects.

\title{
Introducción
}

Del 2015 al 2018, el Instituto de Estudios Interdisciplinarios de la Niñez y la Adolescencia en coordinación con la Oficina de Trabajo Social del Hospital San Vicente de Paúl y la Oficina Regional de Heredia del Patronato Nacional de la Infancia han realizado una serie de procesos socioeducativos con diferentes grupos de adolescentes, madres de la provincia de Heredia, bajo la modalidad de talleres participativos; actividad denominada Redescubriendo a la Persona en la Adolescente Madre.

Se define como objetivo general de la actividad potenciar a la adolescente madre en el redescubrimiento como persona, mediante la participación en espacios de autorreflexión desde una visión de sí misma como persona con derechos.

Se presentan a continuación los objetivos específicos:

1. Lograr en la adolescente madre la identificación de la importancia del redescubrimiento personal, desde una visión de sí misma como persona con derechos 
2. Promover en la adolescente madre los conocimientos, las actitudes y las habilidades que ella posee como estrategias para el redescubrimiento personal desde una visión de sí misma.

3. Retomar sus sentimientos, temores y dificultades como insumos básicos para la interiorización de los nuevos conocimientos, actitudes y habilidades, como estrategias para el redescubrimiento personal

Este artículo surge con el fin de compartir este proceso, su metodología y logros, para resaltar la importancia de apoyar a las adolescentes madres desde la etapa en la que se encuentran, de manera que se favorezca el desarrollo integral en su adolescencia, garantizando el ejercicio pleno de sus derechos, así como su condición de madres.

Redescubriendo a la Persona en la Adolescente Madre es una iniciativa del Instituto de Estudios Interdisciplinarios de la Niñez y la Adolescencia (Ineina), la cual busca propiciar un espacio entre adolescentes madres, en donde, a partir de sus propias vivencias, se traten temas generadores de estrategias en beneficio de su desarrollo.

Para lograr esto, se centra en las adolescentes, más allá de su condición de madres, es decir, se busca rescatar el momento del ciclo vital en el que se encuentren como punto de partida y acompañamiento hacia el logro de una vida con proyectos.

\section{Antecedentes}

En primera instancia, se procede a aclarar que la etapa de la adolescencia comprende de los diez hasta los diecinueve años, según la Organización Mundial de la Salud (2019). Gómez, Molina y Zamberlin (2011), Castillo (2016) y, posteriormente, Papalia y Martorell (2017), la caracterizan como un período de cambios físicos, correlacionados con cambios sociales, cognitivos y psicológicos, los cuales representan para las personas adolescentes un espacio de vida generador de retos, oportunidades, crisis, conflictos y contradicciones.

Además de lo ya mencionado, Krauskopf (2014) afirma que la adolescencia “...es un producto de circunstancias histórico-sociales" (p. 7), también Papalia y Martorell (2017) la consideran como una etapa "que adopta distintas formas en diferentes escenarios sociales, culturales y económicos" (p. 323). Significando la existencia de múltiples adolescencias según contextos y realidades; en resumen, cada persona construye su propia adolescencia.

En esta etapa el proceso se caracteriza por su rápido crecimiento y transformación, en donde las personas adolescentes podrían estar en condiciones de 
riesgo y vulnerabilidad, al tener que enfrentar múltiples situaciones: farmacodependencia, accidentes, fugas, abandono escolar, embarazos, enfermedades de transmisión sexual, violencia contra otras personas y hacia sí mismas, entre otras (Krauskopf, 2014).

Para efectos de este artículo, se desea profundizar en lo referente al embarazo adolescente, considerado como una condición social de múltiples dimensiones. De acuerdo con Salinas, Castro y Fernández (2014), esta condición afecta la salud y la trayectoria escolar de las adolescentes, y en general su proyecto de vida.

De acuerdo con las personas autoras mencionadas, la afectación en su desarrollo educativo incide posteriormente en su inserción laboral, lo que perpetúa la pobreza, el no ejercicio por desconocimiento de los derechos humanos, sexuales y reproductivos, así como la falta de expectativas, frustración, desmotivación, autonomía, participación y ciudadanía de las personas jóvenes, de allí el interés de trabajar este tema.

Es especialmente relevante resaltar datos ofrecidos por el Instituto Nacional de Estadística y Censos (2015), en el caso de Costa Rica existen un total de 349058 adolescentes, de las cuales 7106 entre los 13 y 17 años son madres de una persona infante y 526 son madres por segunda vez, en el mismo rango de edad. En el caso de la provincia de Heredia, el promedio de embarazos de adolescentes madres es de $14,9 \%$ entre los 10 y 17 años.

El embarazo adolescente implica importantes cambios de rol, de golpe la adolescente debe asumir nuevas responsabilidades. Según Krauskopf (2014), la reacción de asumirse y ser madre se potencia en la adolescencia, por la propia etapa del desarrollo que se vive.

Para la autora "asumir los cambios del cuerpo, propios de la adolescencia, al mismo tiempo que las modificaciones propias del embarazo es difícil en sí" (Krauskopf, 2014, p. 143), lo que significa la interacción simultánea de dos procesos complejos, como lo es el cambio de etapa evolutiva y los cambios propios del embarazo, así la persona adolescente debe responsabilizarse de una nueva persona y a su vez seguir con el rol propio de su edad.

\section{Enfoques interactuantes en el proceso de redescubrimiento}

Resulta pertinente contextualizar los diversos enfoques considerados en la ejecución del proceso en su totalidad, los cuales han sido seleccionados según conveniencia con el tema del redescubrimiento de la persona en la adolescente madre. A continuación, se nombran: 
URL: http://www.revistas.una.ac.cr/index.php/dialogo/index

CORREO ELECTRÓNICO: universidadendialogo@una.cr

DOI: http://doi.org/10.15359/udre.11-1.7

Enfoque de derechos: En este caso en particular, se vela por el respeto de los derechos y las obligaciones de las adolescentes madres. Según Pautassi y Royo (2012), se consideran a las niñas o adolescentes como personas con legítimos derechos y responsabilidades, por ende, implican obligaciones para los Estados. Estas responsabilidades deben incidir y reforzar las capacidades y los recursos en la adolescencia, partiendo de los apoyos sociales, como por ejemplo: la familia, la escuela, la comunidad, las instituciones, los sistemas religiosos y culturales.

Enfoque de género: Desde este paradigma, Ulate y Preinfalk (2016) apuntan a la construcción de una sociedad que respeta la diversidad, los derechos humanos, los derechos de la niñez y la adolescencia, al reconocerles como protagonistas de su desarrollo. Por lo tanto, promueve el acceso, uso y control de los recursos, las propiedades, los saberes y la toma de decisiones en condiciones de equidad.

Enfoque biográfico profesional: Su objetivo es "promocionar la aceptación positiva de la identidad sexual y el aprendizaje de conocimientos y habilidades sobre las diversas posibilidades [y condiciones] de la sexualidad en cada etapa evolutiva" (Vargas, Aguilar y Jiménez, 2012, p. 63). Parte del conocimiento científico y del aprendizaje de habilidades (para la toma de decisiones, comunicación, aprendizaje de habilidades sociales), así como de la adquisición de una ética relacional básica en el abordaje de la niñez y la adolescencia como personas con derechos en equidad, con la posibilidad de generar acciones protectoras ante la salud, los riesgos y las vulnerabilidades. De acuerdo con Vargas et al. (2012), uno de los principios clave de este enfoque es el estar libre de estereotipos, prejuicios y ambigüedades, así como reconocer el derecho a un trato digno y respetuoso, en el que se determina la existencia de tantas sexualidades y condiciones como personas, las cuales están circunscritas por las experiencias significativas y autobiográficas.

Enfoque de vulnerabilidad social: Esta perspectiva resulta útil en el abordaje de la niñez y la adolescencia, y desde los grupos o las comunidades a las que pertenecen, que podrían generar múltiples vulnerabilidades y riesgos, determinados por situaciones ambientales, demográficas, socioeconómicas, políticas, jurídicas y culturales. A partir de esta visión interesa identificar los siguientes elementos:

1. Existencia de riesgos externos para las personas menores de edad en su grupo o comunidad

2. Proximidad (entre los riesgos y las vulnerabilidades en su interacción)

3. Posibilidad de evitarlos

4. Capacidad y mecanismos para superar los efectos de esos riesgos

5. Situación final resultante, una vez enfrentadas las consecuencias de la actuación de dichos riesgos (Sánchez y Egea, 2011) 
Los anteriores enfoques funcionan como el paradigma con el cual se busca minimizar los riesgos y sus proximidades, al potenciar a las adolescentes madres desde diferentes conocimientos y actitudes y desde el desarrollo de habilidades, para lo cual se emprende la construcción y sistematización del proceso de redescubrimiento.

\section{Proceso de redescubrimiento}

El proceso de redescubrimiento consiste en un conjunto de fases sucesivas o secuencia de pasos, dispuestos a enfocar a la adolescente madre en su nueva realidad, priorizando en la etapa evolutiva en la que se encuentra.

Este planteamiento propone una serie de experiencias socioeducativas, en las cuales se les facilita a las participantes la búsqueda de nuevas perspectivas de sí mismas, su realidad, desde una visión científica y humanista.

Se resume en tres momentos:

Repensarse como persona desde la visión de sí misma, con sentimientos, temores y dificultades e interiorización para el ejercicio de los derechos humanos, legales y sexuales.

Reinventarse desde los nuevos conocimientos: autoestima, inteligencia emocional, género, relaciones de poder y violencia, alternativas educativas y emprendedurismo.

Reposicionarse a partir de la práctica de nuevas actitudes, habilidades y estilos de vida, relaciones interpersonales, sexualidad integral y vida con proyectos.

\section{Figura 1}

Proceso de redescubrimiento

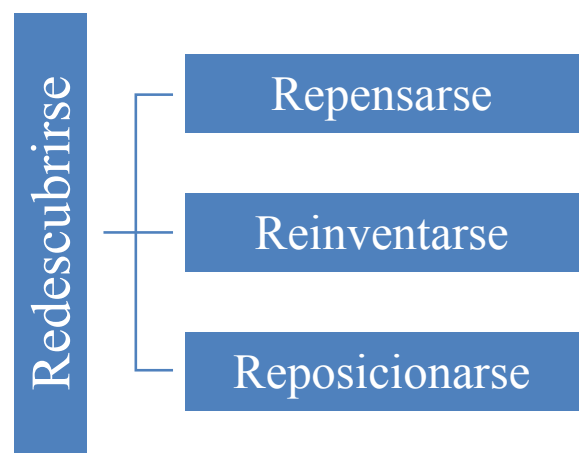

Nota. Elaboración propia 
URL: http://www.revistas.una.ac.cr/index.php/dialogo/index

CORREO ELECTRÓNICO: universidadendialogo@una.cr

DOI: http://doi.org/10.15359/udre.11-1.7

Para facilitar la comprensión de este proceso, se ha procedido a realizar una analogía con respecto al cultivo de una planta: preparar (visión de sí misma), sembrar (conocimientos), producir (actitudes) y consumir (habilidades). A continuación, se detalla:

\section{Figura 2}

Operacionalización desde la analogía del proceso de redescubrimiento

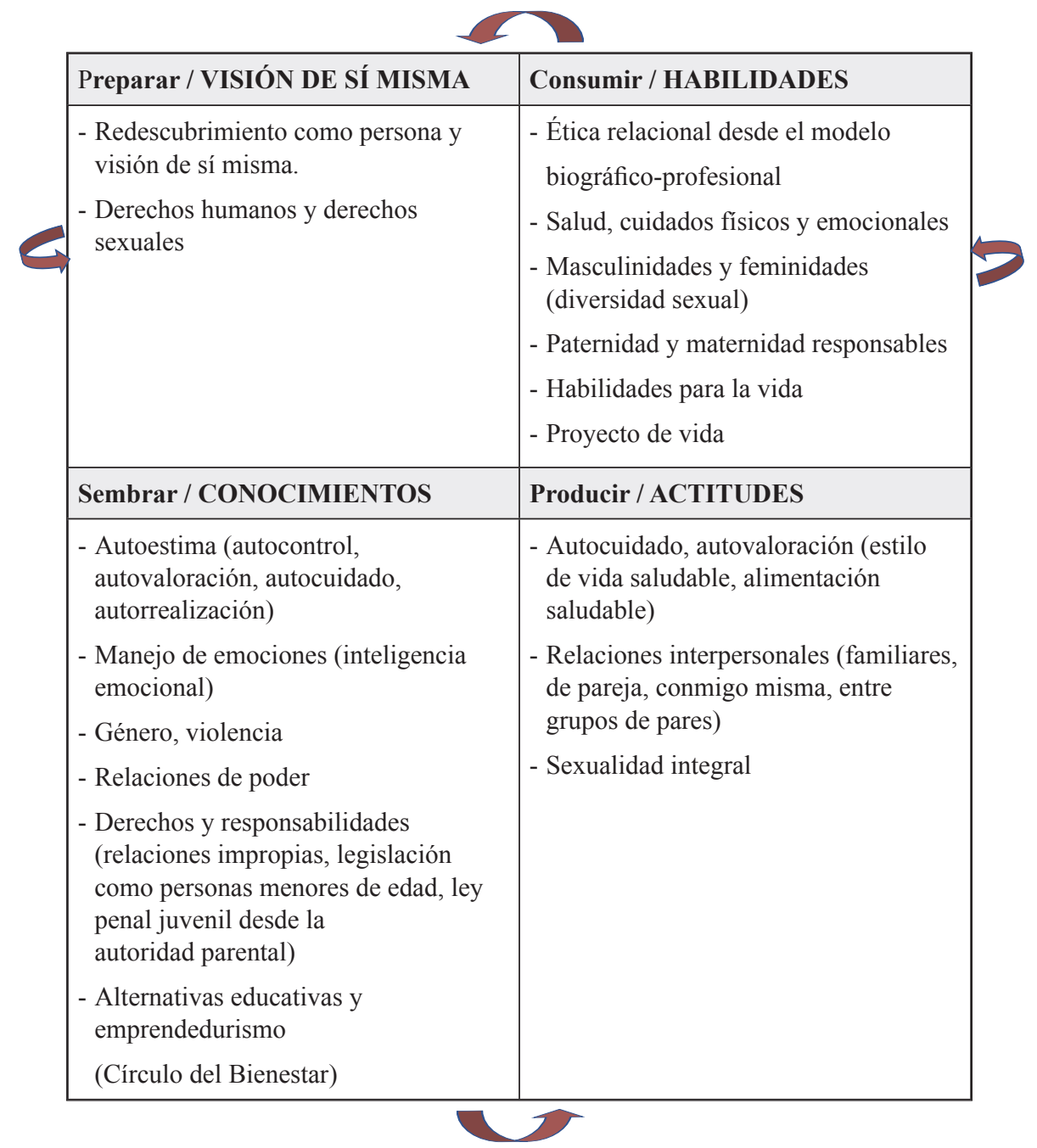

Nota. Elaboración propia 
Así mismo, para profundizar la construcción teórica es pertinente relacionar el redescubrimiento de la persona en la adolescente madre con los niveles de atención propuestos en la Conferencia Alma Ata 1978 (citado por Vignolo, Vacarezza, Álvarez y Sosa, 2011), dándole una naturaleza propia, científica, interdisciplinaria, multidisciplinaria e interinstitucional a este proceso, donde las medidas de atención pueden ir más allá de la esfera médica, al combinar lo teórico y lo pragmático desde las especificidades de cada una de las instituciones participantes, para integrar así cada uno de los niveles de atención con sus respectivas funciones para el beneficio de las adolescentes madres, sus hijos e hijas y familias.

Lo anterior se describe en la siguiente figura:

\section{Figura 3}

Niveles de atención posesionados en la actividad académica
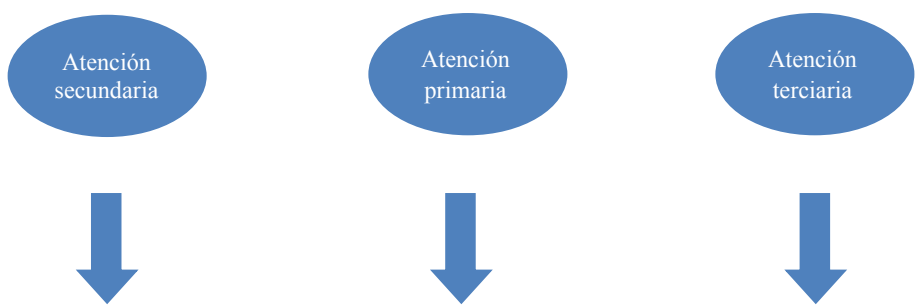

$$
\begin{aligned}
& \text { Desarrollo del } \\
& \text { autoconocimiento, habilidades } \\
& \text { sociales, emocionales, } \\
& \text { derechos, género, sexualidad, } \\
& \text { toma de decisiones y proyecto } \\
& \text { de vida }
\end{aligned}
$$

Nota. Elaboración propia

Atención integral de las adolescentes madres, hijos e hijas y familias en la CCSS y el PANI

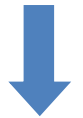

Acceso, uso y control de recursos interinstitucionales, ayudas de becas, atención individual, ofertas educativas y de emprendedurismo, asesoramiento sobre nutrición y métodos anticonceptivos

Según la naturaleza de las instituciones, varían los tipos de respuestas a las adolescentes madres, las cuales se caracterizan por oscilar entre procesos proactivos (visión socioeducativa) y otros más reactivos y asistenciales, con lo cual se visibiliza la oportunidad de impactar de forma sincronizada y efectiva. 
URL: http://www.revistas.una.ac.cr/index.php/dialogo/index

CORREO ELECTRÓNICO: universidadendialogo@una.cr

DOI: http://doi.org/10.15359/udre.11-1.7

\section{Tabla 1}

Tipos de atención

\begin{tabular}{|c|c|c|}
\hline Proactivo & Reactivo & Asistencial \\
\hline $\begin{array}{l}\text { Procesos educativos } \\
\text { que intervienen ante las } \\
\text { vulnerabilidades }\end{array}$ & $\begin{array}{l}\text { Respuestas concretas } \\
\text { hacia los fenómenos } \\
\text { humanos y sociales con } \\
\text { respecto a la adolescente } \\
\text { madre y su condición }\end{array}$ & $\begin{array}{l}\text { Apoyos e instrumentos } \\
\text { orientados a la atención } \\
\text { inmediata según las } \\
\text { necesidades específicas, } \\
\text { de acuerdo con la razón } \\
\text { de ser de las instituciones, } \\
\text { en procura de mejorar } \\
\text { las condiciones, los } \\
\text { conocimientos, las } \\
\text { actitudes y las habilidades }\end{array}$ \\
\hline $\begin{array}{l}\text { Ineina, Departamento de } \\
\text { Trabajo Social, Hospital } \\
\text { San Vicente de Paúl, Cen- } \\
\text { Cinai* y Municipalidad } \\
\text { de Heredia** }\end{array}$ & $\begin{array}{l}\text { Ineina, Departamento de } \\
\text { Trabajo Social, Hospital } \\
\text { San Vicente de Paúl y } \\
\text { Dirección Regional de } \\
\text { Heredia Norte, PANI }\end{array}$ & $\begin{array}{l}\text { Departamento de } \\
\text { Trabajo Social, Hospital } \\
\text { San Vicente de Paúl y } \\
\text { Dirección Regional de } \\
\text { Heredia Norte, PANI }\end{array}$ \\
\hline
\end{tabular}

Nota. $*=$ año 2015; ** $=$ años 2016-2017

\section{Metodología}

El proceso metodológico se apoya en estrategias, técnicas y actividades desde una visión constructivista, en la cual se parte desde los conocimientos y las vivencias previas, compartidas desde cada una de las personas beneficiadas, de forma participativa y democratizada, respetando la edad y la etapa de maduración y logrando asumirse como personas adolescentes.

Es un proceso integral, fundamentalmente socioeducativo, caracterizado por contar con talleres, atención médica, trabajo social (HSPH), asesoría legal (PANI), ofertas educativas (MEP, INA, UNA), emprendedurismo (UNA, Municipalidad de Heredia), que promueven, mediante el redescubrimiento, que la adolescente se mire como persona. 
Se ha realizado de forma anual durante los últimos cuatro años (del 2015 al 2018), con un promedio de catorce sesiones de trabajo, divididas en doce talleres y dos actividades de cierre, donde se generan experiencias de aprendizajes significativos en temáticas promotoras del desarrollo integral de la persona, con las cuales se logra la promoción y protección de sus derechos.

\section{Tabla 2}

Adolescentes madres participantes por año

\begin{tabular}{lllll}
\hline \multicolumn{1}{c}{2015} & 2016 & 2017 & 2018 & $\begin{array}{l}\text { Promedio de } \\
\text { adolescentes madres } \\
\text { beneficiadas }\end{array}$ \\
\hline $\begin{array}{l}\text { Veinticinco } \\
\text { adolescentes } \\
\text { madres }\end{array}$ & $\begin{array}{l}\text { Dieciséis } \\
\text { adolescentes } \\
\text { madres }\end{array}$ & $\begin{array}{l}\text { Catorce } \\
\text { adolescentes } \\
\text { madres }\end{array}$ & $\begin{array}{l}\text { Dieciséis } \\
\text { adolescentes } \\
\text { madres }\end{array}$ & $\begin{array}{l}\text { Setentaiún adolescentes } \\
\text { madres }\end{array}$ \\
\hline
\end{tabular}

Nota. Elaboración propia

Las temáticas abordadas durante los años en mención son:

$\checkmark$ Redescubriendo mi persona

$\checkmark$ Manejo de emociones (inteligencia emocional, resolución asertiva de conflictos)

$\checkmark$ Estilos de vida saludable

$\checkmark$ Salud integral. Alimentación y nutrición de la adolescente: embarazo y período de lactancia. Manipulación de alimentos

$\checkmark$ Modelo biográfico y profesional desde la ética relacional

$\checkmark$ Modelo médico

$\checkmark$ Alimentación infantil. Estimulación y promoción del desarrollo óptimo. Masaje, gateo, estimulación sensorial

$\checkmark$ Disciplina positiva

$\checkmark$ Dinámica familiar (orientando familias)

$\checkmark$ Relaciones de poder, codependencia y violencia

$\checkmark$ Legislación que protege a la adolescente madre.

$\checkmark$ Paternidad responsable

$\checkmark$ Propuestas de derechos humanos 


\author{
$\checkmark$ Replanteamiento del proyecto de vida \\ $\checkmark$ Alternativas educativas: técnicos, ocupaciones y de otra índole \\ $\checkmark$ Emprendedurismo
}

En todas las sesiones, el protagonismo de las adolescentes madres es fundamental a la hora de la construcción y producción de conocimiento y sentimientos, sin olvidar la importancia de los afectos y las emociones involucradas a lo largo del proceso.

Durante la ejecución se administran, tabulan e interpretan diagnósticos, evaluaciones por sesión y entrevistas de seguimiento en línea, las cuales sirven de insumo para la demostración de resultados.

\title{
Resultados
}

Se presentan los resultados obtenidos, cuyos datos se refieren al condensado de cuatro años de implementación del proceso.

De acuerdo con la identificación de la importancia del redescubrimiento personal desde una visión de sí misma como persona con derechos, se puede afirmar el cumplimiento de ochenta y cinco objetivos y contenidos desarrollados durante los cuatro años, correspondientes al promedio de catorce sesiones por año.

Además, se logra ofrecer condiciones determinantes para la permanencia exitosa en los procesos: 850 desayunos, 100 presentes, atención en servicios de medicina, enfermería y trabajo social, en el Hospital San Vicente de Paúl, viáticos y becas por parte del PANI.

Así mismo, se impacta a veintiún comunidades heredianas y setenta y nueve niños y niñas a lo largo de estos años.

Tabla 3

Población infantil beneficiada indirectamente

\begin{tabular}{lllll}
\hline 2015 & 2016 & 2017 & 2018 & $\begin{array}{l}\text { Promedio de } \\
\text { hijos e hijas de } \\
\text { adolescentes madres } \\
\text { que se beneficiaron } \\
\text { indirectamente. }\end{array}$ \\
\hline $\begin{array}{l}\text { Treinta } \\
\text { niños/as } \\
\text { beneficiados/as }\end{array}$ & $\begin{array}{l}\text { Dieciséis } \\
\text { niños/as } \\
\text { beneficiados/as }\end{array}$ & $\begin{array}{l}\text { Dieciséis } \\
\text { niños/as } \\
\text { beneficiados/as }\end{array}$ & $\begin{array}{l}\text { Diecisiete } \\
\text { niños/as } \\
\text { beneficia-dos/as }\end{array}$ & $\begin{array}{l}\text { Setenta y nueve niños/ } \\
\text { as beneficiados/as }\end{array}$ \\
\hline
\end{tabular}

Nota. Elaboración propia 


\section{Tabla 4}

Lugares de procedencia de la población

\begin{tabular}{llll}
\hline \multicolumn{1}{c}{2015} & \multicolumn{1}{c}{2016} & \multicolumn{1}{c}{2017} & \multicolumn{1}{c}{2018} \\
\hline San Rafael, & Guararí, Heredia, & San Rafael, & Heredia, Santa \\
Heredia, San & San Rafael, San & Heredia, Los & Celia, La Milpa, \\
Pablo, San & Joaquín, San & Lagos, Barva, San & Santa Bárbara, \\
Francisco, San & Isidro, San Pablo, & Pedro de Barva, & Santa Lucia, San \\
Isidro, Guayabal, & Santa Bárbara, & San Vicente, & Rafael, San Isidro \\
Cubujuquí, Barva, & Barva, San Pedro & San Isidro & \\
Mercedes Sur, & de Barva, Santa & & \\
Santo Domingo & Lucía de Barva, & & \\
& Los Lagos, & & \\
& Puente Salas, San & & \\
& Francisco, Santa & & \\
& Cecilia y & & \\
& Mercedes Sur & & \\
\hline
\end{tabular}

Nota. Elaboración propia

\section{Construcción de conocimientos obtenidos por las personas informantes:}

De forma cualitativa se obtienen los siguientes resultados desde frases etnográficas mencionadas por las participantes con relación a los objetivos.

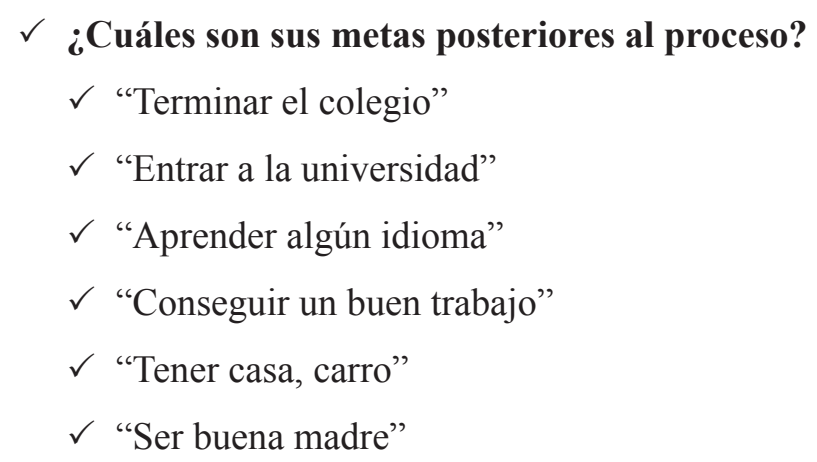

\section{Identificación de la importancia del redescubrimiento:}

"Me enseñó a conocer partes de mí que no conocía" / "Sí pensaba que no tenía oportunidades" / "Reconocernos como personas" / "amor propio" / 
"Ser una nueva madre" / "El respeto que le tiene que dar a uno" / "Saber que puedo continuar adelante, aprender de la experiencia de ser madre adolescente" / "A tener tolerancia, el respeto a los demás y a uno mismo" / "Muchos cambios, saber valorar y valorarme y quererme a mí mismo".

\section{$\checkmark$ Visión de sí misma como persona con derechos:}

"Sí, que ser mamá no es el final de la vida" / "Sí, porque me enseñó a ver que aun siendo mamá podía cumplir mis metas" / "Sí, han recalcado mucho nuestros derechos" / "Sí, nos enseñaron y explicaron los derechos que tenemos" / "Sí dieron a entender que porque soy mamá no significa que no tengo derechos como madre adolescente" / "Sí, porque uno como mujer tiene derechos" / "Derechos y deberes"/ "Sí nos dio a conocer los derechos de los bebes" / "Pensar más en mí como alguien que tiene derechos".

\section{$\checkmark$ Conocimientos, actitudes y habilidades:}

"Me ha ayudado como madre"/ "Sí claro porque te enseñan muchas cosas"/ "Sí claro me enseñó mucho y a saber muchas cosas sobre uno"/ "Respeto hacia los demás" / "Sobre los tipos de violencia" / "Estimulación al bebé" / "Paciencia, mejorar mi carácter" / "Sí cómo poder manejar el carácter" / "Sí a ser una persona diferente y amable con las demás personas" / "Sí trabajamos en autoestima" / "Ser una persona más, con más paciencia y más decidida" / "El respeto y el manejo de carácter" / "A compartir, a llevarme bien con las personas" / "Ser una buena madre" / "Sí la verdad me enseñó mucho".

\section{$\checkmark$ Sentimientos, temores y dificultades:}

"Sí mucho, fue un taller muy especial y enriquecedor" / "No, es complicado después del colegio continuar" / "No sentir vergüenza por haber quedado embarazada tan joven".

Otra forma de obtener datos es comparando el antes y el después del proceso vivido y cómo se relaciona actualmente. En esta evaluación se logra observar los conocimientos, las actitudes y las conductas fortalecidas en el proceso, respaldado con percepciones textuales de las adolescentes madres.

Con respecto a la descripción del funcionamiento de la relación de poder en términos de pareja, en caso de tenerla, se identifican aspectos claros de responsabilidades, relaciones vinculares y afectivas, esfuerzo por construirse, teniendo claro que son personas entre dieciséis y veinte años: 
$\checkmark$ El $80 \%$ indica haber desarrollado un mayor contacto con sus propios sentimientos, para enfrentar sus propios temores.

$\checkmark 90 \%$ utiliza métodos anticonceptivos bajo supervisión del Hospital San Vicente de Paúl.

Finalmente, desde las percepciones de las adolescentes madres, se infiere haber interiorizado aprendizajes con respeto hacia sí mismas y las demás personas, logrando su propio redescubrimiento como adolescentes y madres:

$\checkmark$ "Sí porque me mostró que todos somos diferentes".

$\checkmark$ "Reforzó muchos de mis conocimientos".

$\checkmark$ "No"

$\checkmark$ "Sí claro nos enseñó a ser diferentes".

$\checkmark$ "Sí, mostraba formas de crecer, conocerse y amarse".

$\checkmark$ "Sí, mucho la verdad"

$\checkmark$ "Sí, aprendí a darles respeto".

"Sí, me ayudó mucho a sentirme valiosa por cómo soy".

\section{Discusión}

El proceso de redescubrimiento de la adolescente madre es el planteamiento de un nuevo paradigma en la forma de abordar esta condición, al facilitar la conexión de aprendizajes, habilidades, conocimientos, actitudes y valores en espacios de autorreflexión con la intención de aumentar la visión de sí misma como persona con derechos, de forma procesal, secuencial y actitudinal.

Consiste en cuatro grandes etapas: la primera prepara para profundizar la visión de sí misma, desde los sentimientos y temores; la segunda se centra en sembrar una serie de conocimientos relacionados con el refuerzo del proceso de la autoestima, manejo de emociones, género y poder, alternativas educativas y emprendedurismo, las cuales vienen a empoderarlas como personas adolescentes; vale la pena resaltar que estas dos primeras etapas se centran en "repensarse".

La tercera etapa (reinventarse) se centra en producir actitudes de estilo de vida saludable, relaciones interpersonales y sexualidad integral; finalmente, en la cuarta etapa (reposicionarse) se desarrollan una serie de habilidades, como ética relacional, masculinidades y feminidades, habilidades para la 
vida y vida con proyectos, en las adolescentes madres, las cuales en pocos meses pasan a experimentar de golpe los cambios propios de la etapa de la adolescencia, concatenados con el cambio de rol y las responsabilidades que trae la maternidad, de manera que son niñas criando infantes.

El proceso de redescubrimiento redunda en el aumento del sentido de pertenencia con el cuerpo, la conciencia y la psique, en vinculación con su nueva familia, la comunidad, su autodisciplina, desarrollando y fortaleciendo hábitos, amor propio y autocuidado, lo que implica potenciar a las personas ciudadanas en sus derechos y responsabilidades, para darle así un nuevo sentido a su existencia.

Un detalle a rescatar en el proceso de redescubrimiento consiste en el abordaje de la persona adolescente madre, desde ella misma, con respecto a cuatro enfoques fundamentales (derechos, género, biográfico profesional y el de vulnerabilidad social), los cuales inciden directamente en la visión de mundo de las participantes.

Otro de los aportes consiste en la generación de colaboraciones interinstitucionales (Instituto Nacional de Aprendizaje, Ministerio de Educación Pública, División de Educación para el Trabajo, Municipalidad de Heredia, Cen-Cinai), siendo el Ineina, en representación de la Universidad Nacional, el dinamizador e integrador de macroprocesos, con el fin de procurar la reducción de duplicidades y lograr la distribución adecuada de recursos, dada la dinámica interdisciplinar, transdisciplinar e interinstitucional con poblaciones que mezclan una serie de riesgos y vulnerabilidades.

Es necesario considerar la pertinencia de interrelacionar los niveles de prevención primaria, secundaria y terciaria, dadas las vinculaciones ya mencionadas y debido a la naturaleza de las mismas, las cuales permiten incidir de forma procesal y secuencial en la población meta con enfoques proactivos y reactivos, según las necesidades de las personas participantes.

Con respecto a los logros, se fundamentan en la sistematización de datos y frases etnográficas, citas bibliográficas, observaciones y diálogos colegiados antes y durante la ejecución del proceso.

1. La participación activa y sostenida en cada uno de los años en ejecución consiste en un total de setentaiún adolescentes madres, impactando a veintiún comunidades y setenta y nueve infantes.

2. El proceso de redescubrimiento trae como efecto directo la clarificación de metas a corto, mediano y largo plazo, reforzando el sentido de vida y el de pertenencia. 
3. El proceso en total ha facilitado el redescubrimiento de ellas con una visión integral de sí mismas, como personas con derechos y responsabilidades, encontrando un nuevo sentido a su existencia: repensarse, reinventarse y reposicionarse; con conocimientos, actitudes y habilidades para enfrentar su realidad.

4. El proceso en su potenciación permite poner en contacto a las adolescentes madres desde sus propios sentimientos, temores y dificultades como punto de partida para la toma de decisiones.

5. Las adolescentes madres son potenciadas en el conocimiento, las actitudes y los valores, con un efecto en cadena hacia la pareja, la familia, los hijos, las hijas y la comunidad.

6. Los resultados alcanzados tienen que ver con el aumento del sentido de pertenencia, la vinculación con la comunidad, la autodisciplina y el desarrollo de hábitos.

7. El trabajo interdisciplinario e interinstitucional es posible y permite unificar esfuerzos.

8. Se incide en las adolescentes madres, que son consideradas una población en riesgo y de alta vulnerabilidad.

En esta parte de la discusión resulta pertinente rescatar algunas situaciones y limitaciones: las experiencias en sí han demostrado la posibilidad de trabajar integral, interinstitucional e inter y transdisciplinariamente, pero se requiere un importante esfuerzo para poder lograrlo, implicando a cada una de las partes participantes, con la intención de priorizar, coordinar y negociar para hacerlo realidad, centrándonos en las necesidades y los derechos de las personas a quienes van dirigidos los objetivos y la razón de ser de las instituciones en mención.

Finalmente, es valioso reconocer la importancia de trabajar con las familias (figuras parentales, personas encargadas, parejas), una limitante manifiesta del 2015 al 2018.

\section{Conclusiones}

El proceso de redescubrimiento de la adolescente madre es producto de la interconexión de conocimientos, aprendizajes, habilidades, actitudes y valores y del manejo de la información en espacios de reflexión. La intención de esta metodología consiste en aumentar la visión de sí misma como persona con derechos y obligaciones, de forma procesal, secuencial y actitudinal. 
URL: http://www.revistas.una.ac.cr/index.php/dialogo/index

CORREO ELECTRÓNICO: universidadendialogo@una.cr

DOI: http://doi.org/10.15359/udre.11-1.7

Combina el aumento de condiciones y apoyos, donde las instituciones (Hospital San Vicente de Paúl, IMAS, PANI, Municipalidad de Heredia, Cen- Cinai, INA, MEP), de consuno con el Instituto de Estudios Interdisciplinarios de la Niñez y la Adolescencia, se vinculan al sumar y evitar duplicidades, impactando de forma directa a una de las poblaciones a la que va dirigido su accionar.

El redescubrimiento es un proceso personal fundamentado en múltiples adolescencias, cada una con sus propias trayectorias (historias) y vínculos determinantes, con efectos diversos en la psique humana, ya que cada adolescente, a su forma, en conjunto con sus familias, tendrá que concienciarse de su realidad, propia del contexto en el que se desenvuelve.

La actividad en su totalidad desarrolla conocimientos a través de procesos socioeducativos, ofrece atención integral y promueve el acceso y uso de recursos, con la intención de redescubrirse (repensarse, reinventarse y reposicionarse), pero a cada adolescente le compete generar las transformaciones necesarias para salir o romper los círculos dialelos (condiciones externas), los fenómenos (internos) y las circunstancias (internas y externas), siendo permeados por los otros sistemas (familias, comunidades, centro educativo, sociedad y cultura) y subsistemas (relaciones entre pares y afectivas), que se caracterizan por la interacción.

Resulta necesario profundizar en el concepto de dialelo (del griego diallēlos, 'recíproco'), para resaltar otra de las grandes conclusiones, al comprender que esta estructura lógica de repetición, de reciprocidad (en relación con los otros sistemas y consigo mismo), no necesariamente siempre es vicioso. Esta secuencia de situaciones no se perpetúa, podría ser modificable, en el momento en que se logre identificar y transformar por lo menos una o alguna de ellas para impulsar el cambio, reposicionarse ante nuevos retos y oportunidades.

Por vicio se podría entender uno o varios principios (condiciones, fenómenos y circunstancias) que pueden o no ser crecientes/decrecientes, regulares/ irregulares y que tienen efectos negativos en las personas y en sus vidas.

Un círculo vicioso es un fenómeno compuesto por múltiples causas, consecuencias y/o efectos. Para Sodá, citado por Anónimo (2017), este fenómeno es perjudicial para las personas que se encuentran en dicho círculo, se caracteriza por la repetición constante de uno o más aspectos: condiciones, fenómenos y circunstancias, que mantienen los riesgos y las vulnerabilidades. Cada una con sus propias dinámicas, entretejidas en la cultura predominante (patriarcado), que genera la permanencia y perpetuación en círculo por tiempo 
Revista Universidad en Diálogo • Vol. 11, N. ${ }^{\circ}$ 1, Enero-Junio, 2021 • 179-198

ISSN 2215-2849 • EISSN: 2215-4752

URL: http://www.revistas.una.ac.cr/index.php/dialogo/index CorReo ElECTRÓNICO: universidadendialogo@una.cr DOI: http://doi.org/10.15359/udre.11-1.7

indeterminado, hasta que alguno de los elementos sea modificado. Para ello hay que aumentar el nivel de conciencia en las personas.

Esta actividad académica durante los años de ejecución ha permitido comprender con mayor detalle la estructura lógica del círculo dialelo, compuesto por condiciones, fenómenos y circunstancias construidos con falacias, que afirman de forma equivocada una supuesta secuencia repetitiva, sin principio ni fin, de fenómenos concatenados e indeterminados, los cuales traen como consecuencia perderse en la interacción en una red de otros círculos y sistemas participantes.

Por lo tanto, el proceso de redescubrimiento funciona como un método (razonamiento lógico) en el que las experiencias socioeducativas implementadas permiten incidir en dos factores básicos de vulnerabilidad: la falta de información (roles de género, sobre estimulación de lo coital, conocimiento de la sexualidad en todos sus aspectos) y las vulnerabilidades afectivas (personalidades, historias y experiencias de vida, escenografías familiares: rupturas afectivas y crisis no resueltas, modelaje, disvalores familiares, sentido de pertenencia, inclusión y exclusión de grupos) como elementos disparadores de su embarazo adolescente, descubriendo que todo círculo dialelo tiene un principio y un fin decodificable y, por lo tanto, es posible llevarlo a su fin.

Uno de los aportes fundamentales a compartir desde este paradigma es el aumento de la comprensión de la adolescencia no como una etapa o estadio, sino más bien como la expresión de múltiples adolescencias, caracterizadas por sentimientos, emociones, temores y dificultades propios de cada historia de vida, lo cual implica para las instituciones del Estado una intervención oportuna que obliga a la reinvención de las estrategias vigentes.

Las adolescentes madres combinan factores económicos, socioemocionales, ambientales, de parejas disfuncionales (alcohol y drogas) con efectos en cadena hacia ellas mismas y sus familias, ubicándolas en una posición de vulnerabilidad y amenaza inminente, como factores de riesgo y vulnerabilidad.

Otro aspecto importante a señalar es la identificación de redes de apoyo en sus subsistemas inmediatos, además de las instituciones anteriormente señaladas (Hospital San Vicente de Paúl, IMAS, PANI, Municipalidad de Heredia, CenCinai, INAy MEP), las cuales se consolidan como mesosistemas que interactúan 
URL: http://www.revistas.una.ac.cr/index.php/dialogo/index

CORREO ELECTRÓNICO: universidadendialogo@una.cr

DOI: http://doi.org/10.15359/udre.11-1.7

en beneficio de la salud y la permanencia educativa, como estrategias para la disminución de la reproducción de la pobreza y la potenciación del ser humano en búsqueda de mayores niveles de autorrealización.

Esta actividad académica propone incidir en las trayectorias, la pertenencia con el cuerpo, la afectividad, la disciplina y el autocuidado en sus adolescencias, sus transiciones y en función del desarrollo profesional y de la inserción laboral, aumentando las expectativas, para un mayor manejo de la frustración, aumento de la motivación, la autonomía y la participación ciudadanas.

También, las adolescentes madres indican la posibilidad de haber clarificado metas educativas, laborales, de progreso económico, su maternidad responsable y decisiones de posibles metas profesionales.

Además de lo anterior, este proceso ha contribuido al aumento de la autoconciencia, el autoconocimiento, la autoconfianza, el cumplimiento de metas y el descubrimiento: "la maternidad no es el final", "no se pierden los derechos por efecto de su maternidad", aunado a un sentimiento de "pérdida de vergüenza de la maternidad" y de los sentimientos de culpa.

Vale la pena rescatar el aumento de la conciencia expresada por las adolescentes madres, al identificar la existencia de los "derechos de sus bebés", así como el manejo de su carácter en función del establecimiento de una disciplina positiva.

Este proceso en su totalidad ha permitido demostrar que las vinculaciones interinstitucionales, la interdisciplinariedad y la transdisciplinariedad son posibles en tanto se coloque en primer lugar a las personas, y no a las políticas o los intereses particulares de las gestiones vigentes.

El método científico es el vehículo para que la filosofía y la ciencia del siglo $\mathrm{XX}$ se vean enriquecidas con nuevos saberes del siglo XXI, con efecto en cadena hacia la potenciación de las personas (dimensión psicosocial, espiritual, vocacional, entre otras), la ciudadanía (desde el derecho y las legalidades) y las instituciones (organización de la sociedad).

\section{Agradecimiento}

Un agradecimiento especial a las compañeras y los compañeros de las instituciones que participaron durante los cuatro años de implementación de los procesos. 


\section{Referencias}

Anónimo. (17 de marzo de 2017). El dialelo o círculo vicioso. Altaveu el perquè de tot plegat. https://www.altaveu.com/opinio/el-dialelo-ocirculo-vicioso 2944 102.html

Gómez, P., Molina, R. y Zamberlin, N. (2011). Factores relacionados con el embarazo y la maternidad en menores de 15 años.

Instituto Nacional de Estadística y Censos. (2015). Estadísticas Vitales 2015: población, nacimientos, defunciones, matrimonios. https://www.inec.cr/

Krauskopf, D. (2014). Adolescencia y educación. San José, Costa Rica: Euned.

Organización Mundial de la Salud. (2019). Qué es la adolescencia. https:// www.who.int/topics/adolescent health/es/

Papalia, D. y Martorell, G. (2017). Desarrollo humano (13ª ed.). México: McGraw-Hill/Interamericana Editores.

Pautassi, L. C. y Royo, L. (2012). Enfoque de derechos en las políticas de infancia: indicadores para su medición. https://repositorio.cepal.org/ handle/11362/4044

Salinas, S., Castro, M. y Fernández, C. (2014). Vivencias y relatos sobre el embarazo en adolescentes. https://www.unicef.org/ecuador/embarazo adolescente 5 _0 (2).pdf

Sánchez, G. y Egea, J. C. (Julio-diciembre, 2011). Enfoque de vulnerabilidad social para investigar las desventajas socioambientales. Su aplicación en el estudio de los adultos mayores. Papeles de Población, 17(69), 151-185. https://www.redalyc.org/articulo.oa? $\mathrm{id}=11221117006$

Ulate, C. y Preinfalk, M. (2016). Política para la igualdad y la equidad de género en la Universidad Nacional. http://www.documentos.una.ac.cr/ bitstream/handle/unadocs/7672/002\%20PIEG\%20Y\%20PLAN\%20 DE\%20ACCION.pdf? sequence $=1 \&$ isAllowed $=y$

Fallas Vargas, M. A., Artavia Aguilar, C. y Gamboa Jiménez, A. (2012). Educación sexual: orientadores y orientadoras desde el modelo biográfico y profesional. Revista Electrónica Educare, 16(Especial), 53-71. https://doi.org/10.15359/ree.16-Esp.7

Vignolo, J., Vacarezza, M., Álvarez, C. y Sosa, A. (2011). Niveles de atención, de prevención y atención primaria de la salud. Archivos de Medicina Interna, 33(1), 7-11. http://scielo.edu.uy/scielo. php?script=sci arttext\&pid $=$ S1688-423X2011000100003 